\title{
Partial wave analysis of NN scattering data in chiral EFT
}

\section{Patrick Reinert*}

Ruhr-Universität Bochum, Fakultät für Physik und Astronomie, Institut für Theoretische Physik II, D-44780 Bochum, Germany

E-mail: patrick.reinerterub. de

Nucleon-nucleon (NN) potentials from chiral EFT have entered the precision era with a $\chi^{2} /$ datum $\sim 1$ description of NN scattering data and can provide an alternative to existing partialwave analyses. The framework does not only allow for a model-independent and systematically improvable description of isospin-invariant nuclear forces, but also makes it possible to systematically include and study isospin-breaking effects. We give an overview of the isospin-breaking NN potential up to $\mathrm{N}^{4} \mathrm{LO}$ and give first results for the parameter-free contributions to the two-nucleon system.

The 9th International workshop on Chiral Dynamics 17-21 September 2018

Durham, NC, USA

${ }^{*}$ Speaker. 


\section{Introduction}

Recently, the semi-local momentum-space regularized (SMS) potential up to fifth order ( $\left.\mathrm{N}^{4} \mathrm{LO}\right)$ in chiral EFT has been published [1]. For the SMS potential, we have maintained the idea of a local regularization for the long-range interaction previously employed in Refs. [2,3] while the actual implementation has been carried out in momentum space in anticipation of consistently regularized three-nucleon forces (3NF) and current operators. Additionally, several improvements towards precision physics have been applied to the chiral potentials of Ref. [1]. First, we employ values for the $\pi N$ LECs from the recent Roy-Steiner equation analysis of $\pi N$ scattering [4], which are more precise than those from previous extractions. Further, we have extracted the nucleon-nucleon (NN) LECs directly from NN scattering data instead of relying on the Nijmegen partial-wave analysis [5]. To be more specific, we use the recent 2013 Granada database [6] for our extraction. Motivated by the existence of some high-precision measurement of proton-proton observables which are sensitive to the proper description of F-waves, we added the leading F-wave contact interactions which start to contribute at $\mathrm{N}^{5} \mathrm{LO}$ to the $\mathrm{N}^{4} \mathrm{LO}$ potential. The resulting $\mathrm{N}^{4} \mathrm{LO}^{+}$potential achieves a value of $\chi^{2} /$ datum $\sim 1$ for the description of the $\mathrm{NN}$ scattering database and thus qualifies as a partial-wave analysis.

However, the inclusion of isospin-breaking effects in Ref. [1] has been limited to the pion-mass splitting in the one-pion exchange potential and charge-dependent short-range interactions in the ${ }^{1} S_{0}$ partial wave. While these assumptions about isospin-breaking in the nuclear force have been widely employed and allow for a $\chi^{2} /$ datum $\sim 1$ description for the scattering data, the framework of chiral EFT allows for a systematic incorporation of isospin-breaking effects beyond the ones mentioned above. In this work, we will discuss a complete inclusion of isospin-breaking effects up to $\mathrm{N}^{4} \mathrm{LO}$ in the SMS potential. With the previously achieved precision of $\chi^{2} /$ datum $\sim 1$ we now feel confident to refine our analysis and study the impact of isospin-breaking effects on the extracted phaseshifts.

As a first step in this direction, we will discuss the isospin-breaking potential and its regularization in Section 2. First results for the parameter-free potentials and their contributions to phaseshifts are shown in Section 3. Finally, we conclude in Section 4.

\section{Isospin-breaking $\mathrm{NN}$ interactions}

Isospin symmetry is broken by both strong and electromagnetic effects. On the level of QCD, the fundamental theory underlying chiral EFT, strong isospin-breaking is caused by the mass differences between the up and down quarks while electromagnetic isospin-breaking originates from the different charges of the up and down quarks. In the framework of chiral EFT, where the effective degrees of freedom are nucleons and pions, these isospin-breaking effects among quarks translate to a string of isospin-breaking interaction terms between pions and nucleons in the Lagrangian, which generate e.g. the mass differences between the neutron and the proton as well as between charged and neutral pions. Due to the smallness of these contributions, we do not modify the free Lagrangian mass term to account for the different physical masses of those particles, but rather generate a string of interaction vertices proportional to the strong or electromagnetic contributions to $\delta M_{\pi}^{2}=M_{\pi^{ \pm}}^{2}-M_{\pi_{0}}^{2}$ and $\delta m=m_{p}-m_{n}$, from which the corresponding isospin-breaking diagrams 
can be calculated perturbatively. The relevant expansion parameter for strong isospin-breaking is $\varepsilon M_{\pi}^{2}$ with

$$
\varepsilon \equiv \frac{m_{d}-m_{u}}{m_{d}+m_{u}}
$$

where $m_{u}$ and $m_{d}$ are the masses of the up and down quarks, respectively. On the other hand, electromagnetic isospin-breaking in nuclear forces can be counted in powers of $e^{2}$, where $e$ is the elementary charge. It is convenient to have a unified power counting scheme in only one variable and assign the various isospin-breaking terms to specific orders in the chiral expansion. For this purpose, we adopt the power counting introduced in Ref. [7] and count the additional parameters as

$$
\varepsilon \sim e \sim \frac{Q}{\Lambda_{\chi}}
$$

Additionally, photon loops are counted as

$$
\frac{e^{2}}{(4 \pi)^{2}} \sim \frac{Q^{4}}{\Lambda_{\chi}^{4}}
$$

which accounts for the appearance of the numerically small $1 /(4 \pi)^{2}$ factor in a phenomenological way. In the following, we will distinguish between charge independence-breaking (CIB) and charge symmetry-breaking (CSB) potentials. CIB potentials (also called class II isospin-breaking forces in the classification scheme of Ref. [8]) are proportional to the isospin operator $\tau_{1}^{3} \tau_{2}^{3}$ and maintain charge symmetry, i.e. are identical for proton-proton and neutron-neutron pairs, while CSB potentials do not. CSB potentials include both class III- $\left(\propto \tau_{1}^{3}+\tau_{2}^{3}\right)$ and class IV- $\left(\propto \tau_{1}^{3}-\tau_{2}^{3}\right.$ or $\left.\left(\tau_{1} \times \tau_{2}\right)_{3}\right)$ forces, the former of which conserve total isospin while the latter mix isoscalar and isovector channels. Class IV contributions can be generally assumed to be very small and we neglect them here. In the following, the term "CSB" will always refer to class III forces.

\subsection{One-pion exchange potential}

The general form of the one-pion exchange potential (OPEP) without enforcing the isospin limit is well-known:

$$
\begin{aligned}
& V_{1 \pi}^{p p}=f_{p}^{2} V_{1 \pi}\left(M_{\pi^{0}}\right), \\
& V_{1 \pi}^{n p}=-f_{0}^{2} V_{1 \pi}\left(M_{\pi^{0}}\right)+(-1)^{t+1} 2 f_{c}^{2} V_{1 \pi}\left(M_{\pi^{ \pm}}\right), \\
& V_{1 \pi}^{n n}=f_{n}^{2} V_{1 \pi}\left(M_{\pi^{0}}\right),
\end{aligned}
$$

with $t$ being the two-nucleon total isospin, $f_{0}^{2}=f_{p} f_{n}$ and

$$
V_{1 \pi}\left(M_{i}\right)=-\frac{4 \pi}{M_{\pi^{ \pm}}^{2}} \frac{\vec{\sigma}_{1} \cdot \vec{q} \vec{\sigma}_{2} \cdot \vec{q}}{\vec{q}^{2}+M_{i}^{2}}
$$

Clearly, the CIB due to the pion mass difference is taken into account exactly (i.e. up to infinite order) in eq. (2.4) by employing the correct physical masses of the exchanged pions in the propagators. The one-pion exchange has been the most important model-independent ingredient of the nuclear force since the early days of nuclear physics. Similarly, the CIB due to the pion mass difference in the OPEP yields the dominant long-range isospin-breaking contribution to the 
nuclear force and has been incorporated in all modern high-precision NN potentials and PWAs (e.g. Refs. [5, 6, 10]). While there is universal agreement on the CIB OPEP due to the different physical pion masses, the situation is less clear for the charge-dependence of the OPEP coupling constant. There have been various extractions of $f_{p}^{2}, f_{0}^{2}$ and $f_{c}^{2}$ from both $\pi N$ and $N N$ data over the last decades, see Ref. [11] for a historical overview. One of the most noted results are the determinations of the Nijmegen group, which suggest no charge-dependence of the coupling constant, i.e. $f_{p}^{2}=f_{0}^{2}=f_{c}^{2} \equiv f^{2}$, within the obtained accuracy.

In chiral EFT, the charge-independent coupling constant of the leading order OPEP is given as $f^{2}=M_{\pi^{ \pm}}^{2} g_{A}^{2} /\left(16 \pi F_{\pi}^{2}\right)$. Starting at $\mathrm{N}^{2} \mathrm{LO}$ in our power counting scheme, the OPEP coupling constants receive additional charge-dependent contributions from strong isospin-breaking terms $\propto 2 d_{17}-d_{18}-2 d_{19}$ followed by additional charge-dependent contributions proportional to the electromagnetic LECs $g_{3}$ and $g_{4}$ at $\mathrm{N}^{3} \mathrm{LO}$. However, the values of these LECs are unknown. It seems therefore unavoidable to directly extract $f_{p}^{2}, f_{0}^{2}$ and $f_{c}^{2}$ from NN scattering data in order to study the charge-dependence of the OPEP coupling constants.

At $\mathrm{N}^{3} \mathrm{LO}$, the OPEP receives additional isospin-breaking contributions proportional to $\delta m^{2}$ and $\delta m / m$. The former is given by

$$
V_{1 \pi}^{(4)}=-\delta m^{2} \frac{g_{A}^{2}}{4 F_{\pi}^{2}} \frac{\vec{\sigma}_{1} \cdot \vec{q} \vec{\sigma}_{2} \cdot \vec{q}}{\left(q^{2}+M_{\pi^{ \pm}}^{2}\right)^{2}}\left(\tau_{1} \cdot \tau_{2}-\tau_{1}^{3} \tau_{2}^{3}\right) .
$$

The latter is a class IV isospin-breaking term and thus mixes isoscalar and isovector channels. As mentioned above, we neglect it here, see Ref. [9] for details.

Finally, at $\mathrm{N}^{3} \mathrm{LO}$ we also include the irreducible $\pi \gamma$ exchange potential $[12,13]$

$$
V_{\pi \gamma}(\vec{q})=-\frac{\alpha g_{A}^{2}}{8 \pi F_{\pi}^{2}}\left[\frac{1}{q^{2}}-\frac{\left(M_{\pi^{ \pm}}^{2}-q^{2}\right)^{2}}{q^{4}\left(M_{\pi^{ \pm}}^{2}+q^{2}\right)} \ln \left(1+\frac{q^{2}}{M_{\pi^{ \pm}}^{2}}\right)\right] \vec{\sigma}_{1} \cdot \vec{q} \vec{\sigma}_{2} \cdot \vec{q}\left(\tau_{1} \cdot \tau_{2}-\tau_{1}^{3} \tau_{2}^{3}\right),
$$

which is an electromagnetic correction to the the OPEP.

\subsection{Two-pion exchange potential}

The spin-isospin structure of the isospin-breaking two-pion exchange potential (TPEP) in momentum-space is given as

$$
\begin{aligned}
V(\vec{q}) & =\left(V_{C}^{\mathrm{II}}+V_{S}^{\mathrm{II}} \vec{\sigma}_{1} \cdot \vec{\sigma}_{2}+V_{T}^{\mathrm{II}} \vec{\sigma}_{1} \cdot \vec{q} \vec{\sigma}_{2} \cdot \vec{q}\right)\left(\tau_{1}^{3} \tau_{2}^{3}-\frac{1}{3} \tau_{1} \cdot \tau_{2}\right) \\
& +\left(V_{C}^{\mathrm{III}}+V_{S}^{\mathrm{III}} \vec{\sigma}_{1} \cdot \vec{\sigma}_{2}+V_{T}^{\mathrm{III}} \vec{\sigma}_{1} \cdot \vec{q} \vec{\sigma}_{2} \cdot \vec{q}\right)\left(\tau_{1}^{3}+\tau_{2}^{3}\right) .
\end{aligned}
$$

Here the superscript of the scalar functions $V$ refers to the isospin-breaking classes II and III while the subscripts $C, S, T$ denote central, spin-spin and tensor potential, respectively. Up to $\mathrm{N}^{4} \mathrm{LO}$, terms proportional to the pion mass difference $\delta M_{\pi}^{2}$ are entirely CIB and thus contribute to $V_{X}^{\mathrm{II}}$ while terms proportional to the nucleon mass difference are CSB and hence contribute to $V_{X}^{\text {III }}$ where $X$ collectively denotes $\{C, S, T\}$. See Ref. [9] and Ref. [14] for expressions. ${ }^{1}$

The isospin-breaking TPEP is almost entirely parameter-free in the NN system. Beside the physical nucleon mass difference $\delta m$ we also use the purely strong mass difference $\delta m^{\text {str }}=-2.05$

\footnotetext{
${ }^{1}$ Eq. (2.11) of Ref. [14] corrects a misprint due to a missing factor of $1 / 2$ in eq. (3.52) of Ref. [9].
} 
$\mathrm{MeV}$ [15] to fix these contributions. However, the box-diagram at $\mathrm{N}^{4} \mathrm{LO}$ involves the isospinbreaking $\pi N$ vertex $\propto \beta=\varepsilon M_{\pi}^{2}\left(2 d_{17}-d_{18}-2 d_{19}\right)$ and contributes to

$$
V_{T}^{\mathrm{III}(5)}=-\frac{1}{q^{2}} V_{S}^{\mathrm{III}(5)}=-\frac{g_{A}^{2}}{16 \pi^{2} F_{\pi}^{4}} L(q)\left(\delta m c_{4}+g_{A} \beta\right),
$$

where the logarithmic loop function $L(q)$ is given by

$$
L(q)=\frac{\sqrt{q^{2}+4 M_{\pi}^{2}}}{q} \ln \frac{\sqrt{q^{2}+4 M_{\pi}^{2}}+q}{2 M_{\pi}} .
$$

As discussed in Sec. 2.1, this vertex also contributes to OPEP coupling constants and, following the suggestion of Ref. [9], can be expressed at $\mathrm{N}^{4} \mathrm{LO}$ as

$$
\beta=\frac{f_{p}^{2}-f_{n}^{2}}{8 f_{c}^{2}},
$$

modulo higher order corrections. Thus its contribution to the CSB TPEP can be properly quantified once the charge-dependent OPEP coupling constants have been extracted.

\subsection{Contact interactions}

In addition to the isospin-breaking long-range potential there exist also isospin-breaking contributions to the short-range contact potential. Up to $\mathrm{N}^{4} \mathrm{LO}$, there are both CIB and CSB contributions to the derivative-less contact interactions entering the ${ }^{1} S_{0}$ channel as well as CSB contact terms with two derivatives entering both $\mathrm{S}$ - and $\mathrm{P}$-waves. The partial-wave matrix elements are given as

$$
\begin{aligned}
\left\langle{ }^{1} S_{0}, p p\left|V_{\mathrm{cont}}^{\mathrm{II}+\mathrm{III}}\right|{ }^{1} S_{0}, p p\right\rangle & =\tilde{\beta}_{1 S 0}^{p p}+\beta_{1 S 0}\left(p^{2}+p^{2}\right) \\
\left\langle{ }^{1} S_{0}, n n\left|V_{\mathrm{cont}}^{\mathrm{II}+\mathrm{III}}\right|{ }^{1} S_{0}, n n\right\rangle & =\tilde{\beta}_{1 S 0}^{n n}-\beta_{1 S 0}\left(p^{2}+p^{2}\right) \\
\left\langle{ }^{3} P_{i}, p p\left|V_{\mathrm{cont}}{ }^{\mathrm{III}}\right|{ }^{3} P_{i}, p p\right\rangle & =-\left\langle{ }^{3} P_{i}, n n\left|V_{\mathrm{cont}}^{\mathrm{III}}\right|{ }^{3} P_{i}, n n\right\rangle=\beta_{3 P i} p p^{\prime},
\end{aligned}
$$

with $i=\{1,2,3\}$. The isospin-breaking S-wave contact potential has already been included in the analysis of Ref. [1] while isospin-breaking short-range effects in P-waves have not been taken into account yet.

\subsection{Regularization}

As this work extends the NN potentials of Ref. [1], it is clear that we also employ the same regularization prescription for the new isospin-breaking terms. Before discussing the peculiarities in regularizing these additional terms, we would like to give a brief overview of the semilocal momentum-space regularization introduced in Ref. [1]. It employs a nonlocal regulator for momentum-space matrix elements of contact interactions

$$
V_{\text {cont }}\left(p^{\prime}, p\right) \rightarrow V_{\text {cont }}\left(p^{\prime}, p\right) e^{-\frac{p^{\prime 2}+p^{2}}{\Lambda^{2}}}
$$

as well as a local regulator for the pion-exchange part of the potential, hence the term semilocal. 
In the following, we will elaborate on this local regularization for the long-range potential. Its main idea is to regularize the static pion propagators with a gaussian form factor

$$
\frac{1}{l^{2}+M_{\pi}^{2}} \rightarrow \frac{1}{l^{2}+M_{\pi}^{2}} e^{-\frac{l^{2}+M_{\pi}^{2}}{\Lambda^{2}}}
$$

with $l=|\vec{l}|$ and $\vec{l}$ being the three-momentum of the exchanged pion. This results in properly regularized expressions for the long-range OPEP and TPEP, which, however, still contain an admixture of locally-regularized short-range interactions. We therefore make use of the contact interactions allowed by power counting at a particular chiral order and subtract them from the long-range potential with their coefficients fixed by the requirement that the coordinate-space potentials and as many derivatives thereof as possible vanish at the origin. This procedure yields a clean separation between long-range pion exchanges and short-range contact interactions and leads to a qualitatively similar potential as the coordinate-space regulator employed in Refs. [2, 3].

Regularization of the OPEP in eq. (2.5) is straightforward and leads to

$$
V_{1 \pi}\left(M_{i}\right) \rightarrow V_{1 \pi, \Lambda}\left(M_{i}\right)=-\frac{4 \pi}{M_{\pi^{ \pm}}^{2}}\left(\frac{\vec{\sigma}_{1} \cdot \vec{q} \vec{\sigma}_{2} \cdot \vec{q}}{q^{2}+M_{i}^{2}}+C\left(M_{i}\right) \vec{\sigma}_{1} \cdot \vec{\sigma}_{2}\right) e^{-\frac{q^{2}+M_{i}^{2}}{\Lambda^{2}}}
$$

where $C\left(M_{i}\right)$ is determined by the requirement that the spin-spin part of the coordinate-space potential vanishes as $r \rightarrow 0$. For the TPEP, the scalar functions $V_{2 \pi}(q)$ accompanying the spin-isospin operators can be expressed as spectral integrals over the spectral function $\rho(\mu)=\left.\mathfrak{I}\left(V_{2 \pi}(q)\right)\right|_{q=0^{+}-i \mu}$

$$
V_{2 \pi}(q)=\frac{2}{\pi} \int_{2 M_{\pi}}^{\infty} \mu d \mu \frac{\rho(\mu)}{q^{2}+\mu^{2}}
$$

where, depending on the term at hand, an appropriate number of short-range subtractions have to be performed to render the integration convergent, see Ref. [1] for the individual expressions and corresponding subtraction coefficients discussed above. Regularization of our generic spectral integral in eq. (2.16) yields

$$
V_{2 \pi, \Lambda}(q)=\frac{2}{\pi} \int_{2 M_{\pi}}^{\infty} \mu d \mu \frac{\rho(\mu)}{q^{2}+\mu^{2}} e^{-\frac{q^{2}+\mu^{2}}{2 \Lambda^{2}}}
$$

By expanding the exponentials of either eq. (2.15) or eq. (2.17), it can easily be seen that the regularization does not affect the long-range part of the potential up to any order in the $1 / \Lambda^{2}$ expansion but just corresponds to the addition of an infinite amount of contact interactions whose coefficients are fixed by the form of the regulator.

We now turn to the regularization of the additional isospin-breaking terms discussed in this work. Whenever possible, we apply the regularization prescription outlined above. There are, however, terms which require additional consideration. First, we have to regularize quadratic propagators, i.e. $\left(q^{2}+M_{\pi}^{2}\right)^{-2}$, in e.g. eq. (2.6). These quadratic propagators are not present in the isospin-invariant potential for the adopted "minimal nonlocality" choice $\bar{\beta}_{8}=1 / 4, \bar{\beta}_{9}=-1 / 4$ in the relativistic corrections, see e.g. Ref. [2] for details. Clearly, the $1 / \Lambda^{2}$-term in the gaussian form factor $\exp \left(-\left(q^{2}+M_{\pi}^{2}\right) / \Lambda^{2}\right)$ cannot cancel the quadratic propagator completely and creates an additional long-range contribution. Therefore we adopt the convention

$$
\frac{1}{\left(q^{2}+M_{\pi}^{2}\right)^{2}} \rightarrow\left(\frac{1}{\left(q^{2}+M_{\pi}^{2}\right)^{2}}+\frac{1}{\Lambda^{2}} \frac{1}{q^{2}+M_{\pi}^{2}}\right) e^{-\frac{q^{2}+M_{\pi}^{2}}{\Lambda^{2}}}
$$


which removes the regulator-induced long-range contribution. The additional single-propagator term in eq. (2.18) can, in principle, be induced by a suitably chosen unitary transformation whose generator is proportional to $1 / \Lambda^{2}$.

Another issue arises for the $\pi \gamma$ potential (2.7) and a particular contribution to the CIB TPEP at $\mathrm{N}^{3} \mathrm{LO}$. Usually, contributions to the potential which feature a cut along the imaginary axis in the complex $q$-plane (such as the TPEP) are expressed as spectral integrals which make the $q$ dependence manifest as discussed above. However, these particular terms also feature poles at the start of the cuts which prevents a direct representation in terms of spectral functions. On the other hand, their indefinite integrals with respect to $q^{2}$

$$
F(q)=\int_{0}^{q^{2}} d q^{\prime 2} V\left(q^{\prime}\right)
$$

is in both cases free of poles. Hence we can find $\tilde{\rho}(\mu)=\left.\mathfrak{I}(F(q))\right|_{q=0^{+}-i \mu}$ and differentiate the spectral integral of $F(q)$ to arrive at the once-differentiated spectral integral

$$
V(q)=-\frac{2}{\pi} \int_{n M_{\pi}}^{\infty} \mu d \mu \frac{\tilde{\rho}(\mu)}{\left(q^{2}+\mu^{2}\right)^{2}}
$$

with $n=1(n=2)$ for the $\pi \gamma$ potential (CIB TPEP). Here we again encounter a quadratic propagator inside the spectral integral whose regularization is completely analogous to eq. (2.18) and leads to

$$
V_{\Lambda}(q)=-\frac{2}{\pi} \int_{n M_{\pi}}^{\infty} \mu d \mu \tilde{\rho}(\mu)\left(\frac{1}{\left(q^{2}+\mu^{2}\right)^{2}}+\frac{1}{n \Lambda^{2}} \frac{1}{q^{2}+\mu^{2}}\right) e^{-\frac{q^{2}+\mu^{2}}{n \Lambda^{2}}}
$$

\section{Results for parameter-free contributions}

Let us start by looking at the size of the different contributions to the isospin-breaking TPEP. Fig. 1 shows the regularized scalar functions $V_{C, \Lambda}(r), V_{S, \Lambda}(r)$ and $V_{T, \Lambda}(r)$ accompanying the corresponding spin operators in coordinate space for $\Lambda=450 \mathrm{MeV}$. See e.g. Appendix B of Ref. [1] for explicit formulas for the Fourier transformation from eq. (2.8) to coordinate-space. Considering the CIB TPEP, $V_{C}^{\mathrm{II}}(r)$ only receives contributions at $\mathrm{N}^{3} \mathrm{LO}$ while contributions to $V_{S}^{\mathrm{II}}(r)$ and $V_{T}^{\mathrm{II}}(r)$ start at $\mathrm{N}^{4} \mathrm{LO}$. These spin-spin and tensor terms are proportional to $\delta M_{\pi}^{2}$ and the numerical large LEC $c_{4}$ and are clearly the largest contribution from the isospin-breaking TPEP reaching up to 300 $\mathrm{keV}$ for $V_{T, \Lambda}^{\mathrm{II}}(r)$ at $\Lambda=450 \mathrm{MeV}$. This has to be compared to the isospin-invariant TPEP which is of the order of $\sim 2-12 \mathrm{MeV}$, see Fig. 6 and Fig. 7 of Ref [1] for the isospin-invariant TPEP in coordinate-space. Looking now at the CSB TPEP, we see that the subleading contribution to $V_{C}^{\mathrm{III}}(r)$ at $\mathrm{N}^{4} \mathrm{LO}$ is notably larger than the leading one at $\mathrm{N}^{3} \mathrm{LO}$. This is not surprising and indeed the same pattern also arises for the leading and subleading isospin-invariant TPEP. It is caused by the numerically large values of the subleading $\pi N$ LECs $c_{3}$ and $c_{4}$ which are well-understood in terms of resonance saturation [16]. We note that $V_{S, \Lambda}^{\mathrm{III}}(r)$ and $V_{T, \Lambda}^{\mathrm{III}}(r)$ as shown in Fig. 1 have been obtained by evaluating the regularized form of eq. (2.9) with $\beta=0$ and that their final contribution can only be evaluated once $\beta$ is known. Further, when comparing the sizes of the CIB and CSB TPEP as shown in Fig. 1 one has to keep in mind that the CSB TPEP receives an additional factor of \pm 2 when evaluated for a proton-proton or neutron-neutron pair, respectively, due to the isospin operator $\tau_{1}^{3}+\tau_{2}^{3}$. 

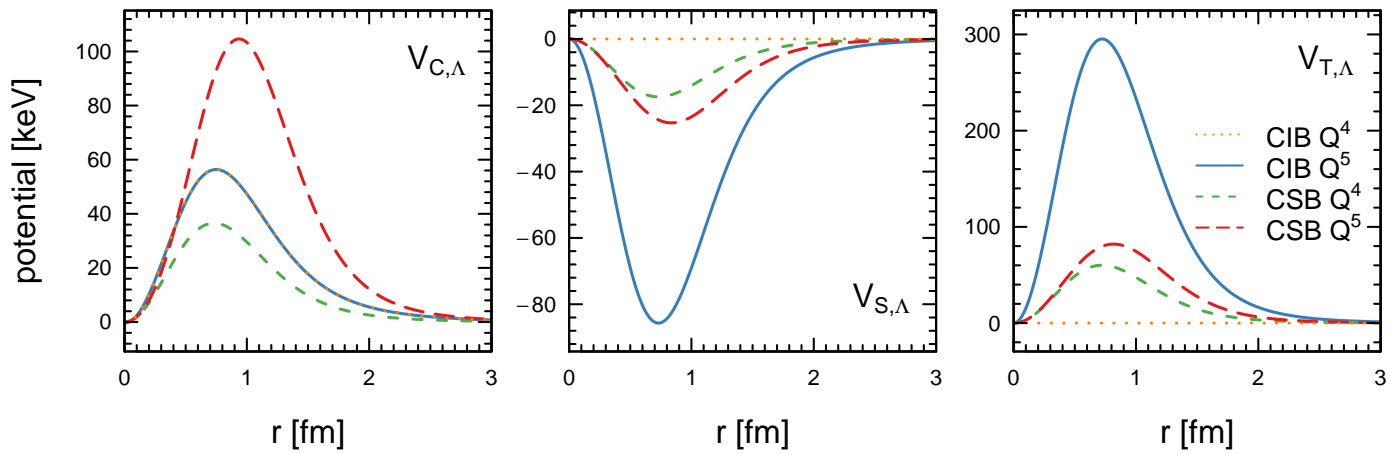

Figure 1: Scalar functions of the regularized isospin-breaking TPEP in coordinate space for $\Lambda=450 \mathrm{MeV}$. Dotted ochre and solid blue lines refer to the CIB TPEP $\left(V_{X}^{\mathrm{II}}\right)$ at $\mathrm{N}^{3} \mathrm{LO}$ and $\mathrm{N}^{4} \mathrm{LO}$ while dashed green and long-dashed red lines refer to the CSB TPEP $\left(V_{X}^{\mathrm{III}}\right)$ at $\mathrm{N}^{3} \mathrm{LO}$ and $\mathrm{N}^{4} \mathrm{LO}$.

Due to our convention of subtracting out the short-range components via the freedom in available contact interactions, all potentials in Fig. 1 vanish at the origin by construction. This is also true for other terms in the potential with the exception of the $\pi \gamma$ exchange potential. Here, no subtractions can be performed based on our power-counting which leads to a non-vanishing spin-spin potential at the origin in coordinate-space as shown in Fig. 2. One can see that the $\pi \gamma$ exchange potential is generally small. We also show the cutoff variation between $\Lambda=400-550 \mathrm{MeV}$. As can be seen, the spin-spin part is fairly cutoff-independent for $r>1 \mathrm{fm}$ while the tensor part reaches cutoff-independence for about $r>2 \mathrm{fm}$.

How much do these new isospin-breaking terms affect the partial-wave analysis? As a first step, we consider the impact of the parameter-free terms on the phaseshifts. Fig. 3 shows the individual contributions of the CIB OPEP and the $\pi \gamma$ potential as well as the CIB and CSB TPEP to the difference $\delta_{n p}-\delta_{p p}$ between isovector phases in the neutron-proton and proton-proton system.
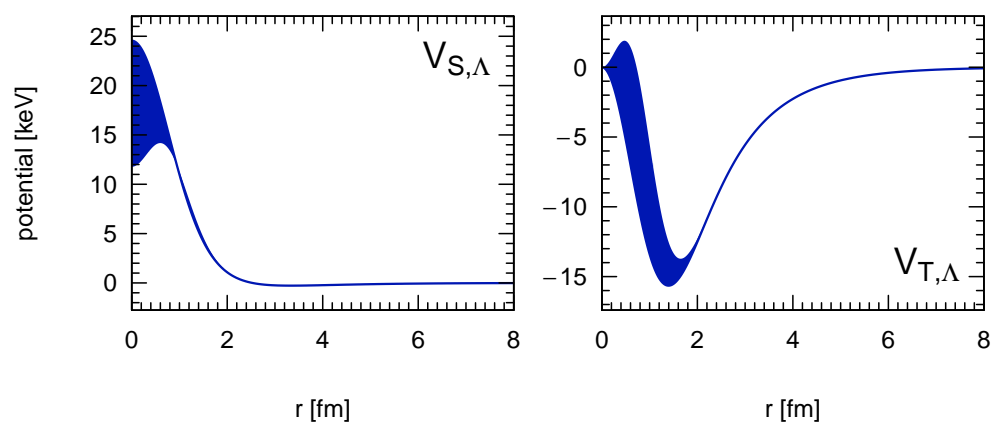

Figure 2: Scalar functions for the spin-spin and tensor part of the regularized $\pi \gamma$ exchange potential in coordinate-space. Dark blue bands represent the cutoff variation of the $\pi \gamma$ exchange potential for $\Lambda=$ $400-550 \mathrm{MeV}$. 

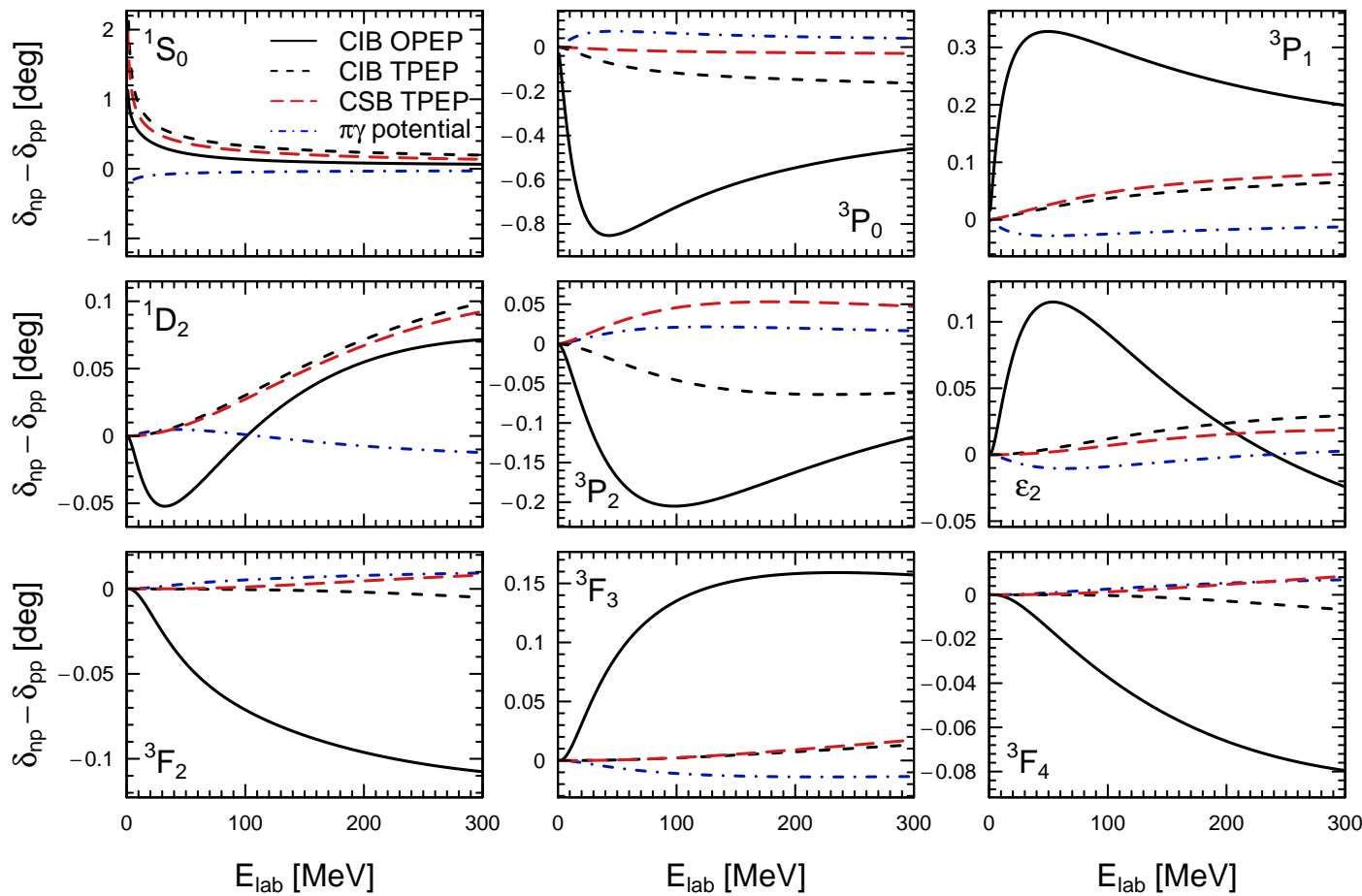

Figure 3: Individual contributions of isospin-breaking terms to np - pp phaseshift difference $\delta_{n p}-\delta_{p p}$ with respect to spherical bessel functions.

For the $p p$ system, the Coulomb potential has been set to zero and phases have been calculated with respect to spherical bessel functions. As can been seen, the CIB OPEP is the dominant contribution in most partial waves. This is especially true for higher partial waves where the long-range nature of the OPEP leads to a slower decrease with increasing partial waves compared to the shorter-range TPEP. Strictly speaking, the CIB OPEP shown here includes the well known isospin-breaking effect due to the different pion masses as well as the term in eq. (2.6), the latter of which is, however, negligible on the scale of the figure. For S-, P- and D-waves the TPEP yields sizable contributions to the phaseshift difference. It is interesting to see, that the CIB and CSB TPEP corrections to the $\delta_{n p}-\delta_{p p}$ are often comparable in size while one would naively expect the CIB ones to be larger in the hierarchy among isospin-breaking classes. Finally, the impact of the $\pi \gamma$ exchange potential is small but non-negligible. It always reduces the phaseshift difference due to the CIB OPEP by a small amount.

It has to be stressed that the results above represent only the parameter-free (in the NN system) contributions and are thus incomplete. Our preliminary studies indicate that further corrections to $\delta_{n p}-\delta_{p p}$ due to the charge-dependence of the OPEP coupling constants could reach up to $50 \%$ of the size of those due to the CIB OPEP at intermediate energies of $E_{\text {lab }}=100-200 \mathrm{MeV}$. Likewise, the isospin-breaking contact interactions seem to be large in ${ }^{3} P_{1}$ and ${ }^{3} P_{2}$. Work to extract these contributions from the NN system is in progress. 


\section{Conclusions}

In these proceedings, we discussed the isospin-breaking potential from chiral EFT up to ${ }^{4} \mathrm{LO}$ in the employed unified power counting scheme. The new terms have been consistently regularized for the inclusion in the semilocal momentum-space potentials of Ref. [1] and we have detailed the extension of the SMS regulator to newly appearing structures in the isospin-breaking potential. Finally, the size of the parameter-free isospin-breaking potential and its impact on the phaseshifts was discussed. Clearly, the remaining unknown effects due to the charge-dependence of the OPEP coupling constants and isospin-breaking contact interactions in P-waves have to be determined for a complete and conclusive analysis of isospin-breaking in the two-nucleon system. Work along these lines is in progress.

\section{Acknowledgments}

This work was supported in part by DFG (SFB/TR 110, "Symmetries and the Emergence of Structure in QCD") and the BMBF (Grant No. 05P18PCFP1).

\section{References}

[1] P. Reinert, H. Krebs and E. Epelbaum, Eur. Phys. J. A 54, no. 5, 86 (2018).

[2] E. Epelbaum, H. Krebs and U. G. Meißner, Eur. Phys. J. A 51, 53 (2015).

[3] E. Epelbaum, H. Krebs and U. G. Meißner, Phys. Rev. Lett. 115, 122301 (2015).

[4] M. Hoferichter, J. Ruiz de Elvira, B. Kubis and U. G. Meißner, Phys. Rev. Lett. 115, no. 19, 192301 (2015).

[5] V. G. J. Stoks, R. A. M. Klomp, M. C. M. Rentmeester and J. J. de Swart, Phys. Rev. C 48, 792 (1993). doi:10.1103/PhysRevC.48.792

[6] R. Navarro Pérez, J. E. Amaro and E. Ruiz Arriola, Phys. Rev. C 88, no. 6, 064002 (2013) Erratum: [Phys. Rev. C 91, no. 2, 029901 (2015)].

[7] E. Epelbaum, W. Glöckle and U. G. Meißner, Nucl. Phys. A 747, 362 (2005).

[8] E. M. Henley and G. A. Miller, in Mesons and Nuclei, edited by M. Rho and G. E. Brown (North Holland, Amsterdam, 1979), Vol. I, p. 405

[9] E. Epelbaum and U. G. Meißner, Phys. Rev. C 72, 044001 (2005).

[10] V. G. J. Stoks, R. A. M. Klomp, C. P. F. Terheggen and J. J. de Swart, Phys. Rev. C 49, 2950 (1994).

[11] E. Matsinos, arXiv:1901.01204 [nucl-th].

[12] U. van Kolck, M. C. M. Rentmeester, J. L. Friar, J. T. Goldman and J. J. de Swart, Phys. Rev. Lett. 80, 4386 (1998).

[13] N. Kaiser, Phys. Rev. C 73, 044001 (2006).

[14] E. Epelbaum, H. Krebs and U. G. Meißner, Phys. Rev. C 77, 034006 (2008).

[15] J. Gasser and H. Leutwyler, Phys. Rept. 87, 77 (1982).

[16] V. Bernard, N. Kaiser and U. G. Meissner, Nucl. Phys. A 615, 483 (1997) doi:10.1016/S0375-9474(97)00021-3 [hep-ph/9611253]. 